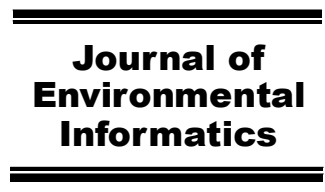

www.iseis.org/jei

\title{
Space-Time Metric Determination in Environmental Modeling
}

\author{
G. Christakos ${ }^{1, *}$, J. M. Angulo ${ }^{2}$, H. L. $\mathrm{Yu}^{3}$, and J. $\mathrm{Wu}^{1, *}$ \\ ${ }^{1}$ Department of Geography, San Diego State University, San Diego, CA, USA and Institue of Island and Coastal Ecosystems, \\ Ocean College, Zhejiang University, Hangzhou, China \\ ${ }^{2}$ Department of Statistics and Operations Research, University of Granada, Granada, Spain \\ ${ }^{3}$ Bioengineering Department, National Taiwan University, Taipei, Taiwan
}

Received 6 December 2015; revised 22 October 2016; accepted 29 November 2016; published online July 12017

\begin{abstract}
Physical space-time metrics are used in environmental modeling to define "distance" between points in the space-time domain of a physical attribute (contaminant concentration, exposure, temperature etc.). Assessing a space-time metric is often a considerably more complicated affair than assessing a purely spatial metric. This is because the physical space-time metric suggests a certain concept of distance that blends space and time to make space-time, but at the same time, it views time as a dissimilar quantity. In this work, the determination of space-time metrics takes advantage of the strong links between the physical characteristics of the real-world attribute and the geometrical features of the composite space-time domain within which the attribute occurs. Via physical law an explicit connection is established between attribute's space-time dependence structure (represented by the covariance function) and attribute's domain geometry (expressed by the metric coefficients). The derived physical geometry equation can be solved for the metric coefficients. The solution depends not only on the form of the physical law, but also on the boundary/initial conditions and the randomness sources. The proposed approach turns metric coefficients into physically meaningful parameters, allowing better understanding of the space-time characteristics than the ad hoc and arbitrary metric selection in purely technical terms.
\end{abstract}

Keywords: environment, modeling, space-time, metric, stochastics, covariance, physical law

\section{Introduction}

It is widely recognized in the environmental literature that a metric structure that involves theoretical and empirical facts about the investigated attribute (contaminant concentration, exposure, temperature, pressure etc.) is required in order to gain a higher level understanding of space-time in environmental modeling (Arkin and Ardanuy, 1989; Jin et al., 2014; Reyes and Serre, 2014). All quantitative tools assessing the strength of space-time dependence and correlation in environmental studies (like covariance and variogram tools) are functions of the metric. This means that these tools can be valid for one kind of space-time metric but invalid for some others, and, also, the space-time metric has a direct effect on environmental applications based on these tools, like space-time variability assessment and mapping (e.g., Kriging, Kyriakidis and Journel, 1999). Most of the existing studies of space-time environmental attributes consider space and time separately (Stein et al., 1998; Le and Zidek, 2006). However, this viewpoint is often problematic, since even in a classical Newtonian framework the

* Corresponding author. Tel: +86 580 2092306; fax: +86 5802092891 E-mail address: gchristakos@zju.edu.cn(G.Christakos), jw67@zju.edu.cn (Jiaping Wu).

SSN: 1726-2135 print/1684-8799 online

(C) 2017 ISEIS All rights reserved. doi: 10.3808/jei.201700365 simplistic space-time metric consisting of two separate components -a purely spatial (Euclidean) component with constant coefficients and a time component- may be convenient but, nevertheless, inadequate. One should keep in mind that almost all data in applied sciences are closely interrelated both in space and time, and that it is this space-time interrelation that, both, it is at the heart of the physical laws of space-time change, and it also allows the representation of the space-time variation of a physical phenomenon from a limited number of observations. Furthermore, a sound physical metric may be constrained by the physical invariance transformations it must satisfy in order to be meaningful (Christakos and Hristopulos, 1998; Hadsell and Hansen 1999; Carroll 2004). The above comments concerning composite space-time analysis are valid in studies in a wide range of disciplines, like earth and atmospheric sciences, meteorology, public health and space-time epidemiology.

In view of the above considerations, in this work the spacetime metric is considered as an integrated whole and its coefficients are found as the solutions of the set of equations derived so that they establish an explicit link between physical knowledge of the environmental attribute's space-time dependence structure (expressed by its covariance function) and the geometry of the attribute domain (expressed by its composite space-time metric). In a nutshell, instead of arbitrarily selecting a space-time metric for the phenomenon of interest (as is commonly done in most cases of environmental modeling practice), the proposed approach suggests allowing the physical law to 
Table 1. Special Cases of the Metric of Equation (2) in $R^{n} \times T$.

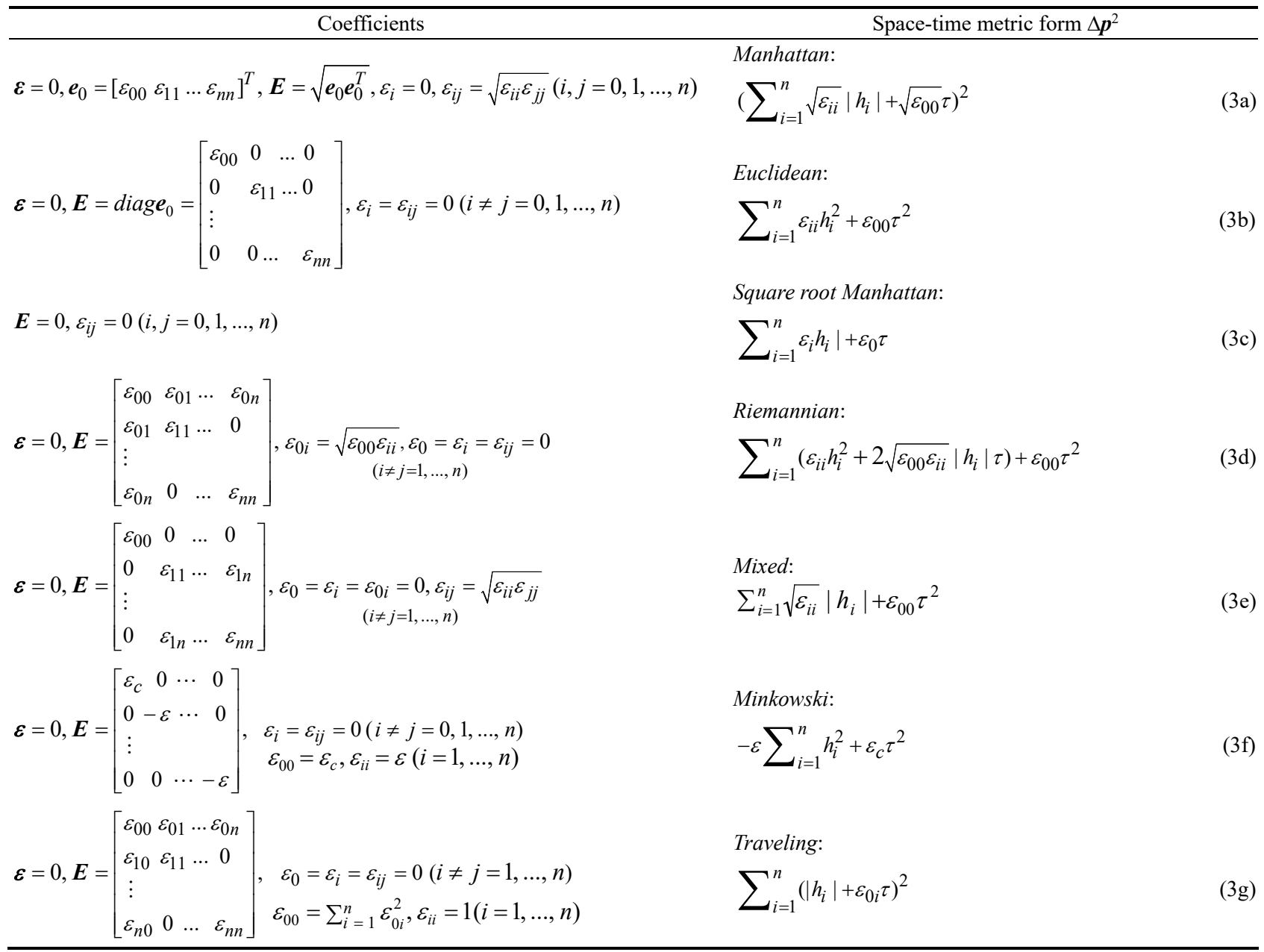

reveal the metric form. The metric is generally non-separable in space and time, and it depends on the physical law of change, the associated boundary/initial conditions, and the randomness sources of the environmental phenomenon. Knowing the metric in closed form, the elucidation of its physical properties is based on the features of the phenomenon under consideration, e.g., the interpretation of the metric coefficients and their relationships relies on the corresponding physical law.

\section{Methods}

\subsection{Space-Time Metrics}

Several kinds of metrics or "distances" (Euclidean and non-Euclidean, have been considered in the environmental literature (e.g., Turcotte 1997; Christakos 2000; Christakos et al., 2000; Billings et al., 2002; Frei, 2014; Lin et al., 2015). For the purposes of the present study we introduce the following definition of a general space-time metric in the $n$-dimensional space $\times$ time, $R^{n} \times T$ :

$$
\Delta \boldsymbol{p}^{2}=\breve{\boldsymbol{h}}^{T} \breve{\boldsymbol{E} \boldsymbol{h}}
$$

where in vector $\breve{\boldsymbol{h}}=\left[\boldsymbol{h}^{T}, 1\right]^{T}=\left[h_{0} h_{1} \ldots h_{n} 1\right]^{T}$, the $h_{1} \ldots h_{n}$ denote space lag and the $h_{0}=\tau$ time lag, and:

$\breve{\mathbf{E}}=\left[\begin{array}{cccc}\varepsilon_{00} & \varepsilon_{01} \ldots \varepsilon_{0 n} & \frac{1}{2} \varepsilon_{0} \\ \varepsilon_{10} & \varepsilon_{11} \ldots \varepsilon_{1 n} & \frac{1}{2} \varepsilon_{1} \\ \vdots & \\ \varepsilon_{n 0} & \varepsilon_{n 1} \ldots \varepsilon_{n n} \frac{1}{2} \varepsilon_{n} \\ \frac{1}{2} \varepsilon_{0} & \frac{1}{2} \varepsilon_{1} \ldots \frac{1}{2} \varepsilon_{n} 0\end{array}\right]$

Knowing the metric in a closed form, elucidation of its properties is based on the phenomenon (Christakos and Hristopulos, 1998; Hadsell and Hansen, 1999; Carroll 2004). The metric of Equation (1) defines the connection between any two points in the space-time domain. For subsequent analytical manipulations it may be more convenient to rewrite Equation (1) as:

$$
\Delta \boldsymbol{p}^{2}=\boldsymbol{h}^{T}(\boldsymbol{E} \boldsymbol{h}+\boldsymbol{\varepsilon})
$$

where $\boldsymbol{h}=\left[\begin{array}{llll}h_{0} & h_{1} & \ldots & h_{n}\end{array}\right]^{T}\left(h_{0}=\tau\right)$, and 


$$
\begin{aligned}
& \boldsymbol{E}=\left[\begin{array}{cccc}
\varepsilon_{00} & \varepsilon_{01} & \ldots & \varepsilon_{0 n} \\
\vdots & & \\
\varepsilon_{n 0} & \varepsilon_{n 1} & \ldots & \varepsilon_{n n}
\end{array}\right], \\
& \boldsymbol{\varepsilon}=\left[\begin{array}{lllll}
\varepsilon_{0} & \varepsilon_{1} & \ldots & \varepsilon_{n}
\end{array}\right]^{T}
\end{aligned}
$$

are matrix and vector, respectively, of metric coefficients. The metric coefficients $\boldsymbol{E}$ and $\boldsymbol{\varepsilon}$ may be themselves functions of the space-time coordinates or they may be functions of physical quantities as required by the real world situation. The metric expression of Equation (2) is quite general. For example, in some cases a metric may be defined for convenience in terms of the absolute coordinate distances $|\boldsymbol{h}|$, instead of the signed lags $\boldsymbol{h}$. Indeed, if we replace $\boldsymbol{E}$ and $\boldsymbol{\varepsilon}$ in Equation (2) by $\boldsymbol{E}_{\boldsymbol{A}}=$ $\boldsymbol{\Lambda}(\boldsymbol{h}) \boldsymbol{E} \boldsymbol{\Lambda}(\boldsymbol{h})$ and $\boldsymbol{\varepsilon}_{\boldsymbol{\Lambda}}=\boldsymbol{\Lambda}(\boldsymbol{h}) \boldsymbol{\varepsilon}$, where $\boldsymbol{\Lambda}(\boldsymbol{h})$ is an $(n+1) \times(n+1)$ diagonal matrix with elements $\operatorname{sign}\left(h_{i}\right)= \pm 1$ or $0(i=0,1, \ldots, \mathrm{n})$ depending on whether $h_{i}$ is positive, negative or null, Equation (2) gives the metric expressions $\Delta \boldsymbol{p}^{2}=|\boldsymbol{h}|^{T}(\boldsymbol{E}|\boldsymbol{h}|+\boldsymbol{\varepsilon})$, or $\Delta \boldsymbol{p}^{2}=$ $\boldsymbol{h}^{\mathrm{T}} \boldsymbol{E} \boldsymbol{h}+|\boldsymbol{h}|^{T} \boldsymbol{\varepsilon}$, where $|\boldsymbol{h}|=\left[\left|h_{0}\right|,\left|h_{1}\right|, \ldots,\left|h_{n}\right|\right]^{T}$. Metric symmetry is readily satisfied for space- and time-independent $\boldsymbol{E}$ and $\boldsymbol{\varepsilon}$, and also for space- and time-dependent $\boldsymbol{E}$ and $\boldsymbol{\varepsilon}$ assuming that $\boldsymbol{E}(\boldsymbol{h})=\boldsymbol{E}(-\boldsymbol{h})$ and $\boldsymbol{\varepsilon}(\boldsymbol{h})=\boldsymbol{\varepsilon}(-\boldsymbol{h})$. Equation (2) includes the metrics defined in terms of $|\boldsymbol{h}|$ (like the Manhattan metric in Table 1 ), as far as the matrix $\boldsymbol{E}$ and vector $\boldsymbol{\varepsilon}$ implicitly involve, in these cases, the change to positive sign in the negative coordinates of $\boldsymbol{h}$ (by means of $\boldsymbol{\Lambda}(\boldsymbol{h})$ ).

Although one may usually choose a space-time coordinate basis so that the inner product that defines the metric is diagonal and normalized (Euclidean or flat spaces), in certain cases it may be reasonable to choose a different coordinate basis. As far as real world science is concerned, it is important to determine physical metrics that actually represent or stem from $\mathrm{Na}-$ ture and from experimental evidence rather than simply imposed by pure mathematics often involving convenient conditions not based necessarily upon real-world experience. Accordingly, a number of metrics or "distances" (Euclidean and non-Euclidean), have been considered in the applied sciences literature (Turcotte, 1997; Christakos and Papanicolaou, 2000; Billings et al., 2002; Curriero, 2006; Lloyd, 2010; Lin et al., 2015). In this context, Equation (2) is a general metric that includes other common space-time metrics used in environmental sciences as its special cases. Particular cases of the metric of Equation (2) are listed in Table $1(\tau>0)$. Although the metric coefficients are generally space- and/or time-dependent, in many cases of practical interest independency may apply. Herein, for simplicity the "|.|" is dropped when the meaning of $\Delta \boldsymbol{p}$ is obvious from the context.

Based on the space-time metric formulation above, we introduce the metric differential formulas (MDF) expressing metric change in the composite space-time domain:

$$
\frac{\partial \Delta \boldsymbol{p}}{\partial \boldsymbol{h}}=\left(\boldsymbol{h}^{T} \boldsymbol{E}+\frac{1}{2} \boldsymbol{\varepsilon}^{T}\right) \Delta \boldsymbol{p}^{-1}
$$

$\frac{\partial \Delta \boldsymbol{p}^{-1}}{\partial \boldsymbol{h}}=-\frac{\partial \Delta \boldsymbol{p}}{\partial \boldsymbol{h}} \Delta \boldsymbol{p}^{-2}$

$$
\frac{\partial^{2} \Delta \boldsymbol{p}}{\partial \boldsymbol{h}^{T} \partial \boldsymbol{h}}=\left(\boldsymbol{E}-\frac{\partial \Delta \boldsymbol{p}}{\partial \boldsymbol{h}^{T}} \frac{\partial \Delta \boldsymbol{p}}{\partial \boldsymbol{h}}\right) \Delta \boldsymbol{p}^{-1}
$$

where $\partial \boldsymbol{h}^{T}=\left[\partial h_{0}=\partial \tau \partial h_{1} \ldots \partial h_{n}\right]$. Space- and time-dependent metric coefficients would be expressed in terms of standard analytic functions (e.g., polynomials) of the corresponding coordinates.

\subsection{Covariance Differential Formulas}

Covariance functions are important stochastic modeling tools that are used in environmental sciences to represent the space-time dependency structure of physical attributes in conditions of uncertainty (Purser et al., 2003; Lloyd, 2010; Tartakovsky and Broyda, 2011; He et al., 2014). In the covariance differential formulas (CDF) introduced below, for any subset of (possibly repeated) indexes $J=\left\{j_{1}, \ldots, j_{v}\right\}$, we let $\partial_{J}^{v}=\partial^{v} /$ $\partial h_{j_{1}} \ldots \partial h_{j_{v}}$ and introduce the class $\boldsymbol{P}_{m: k}$ of partitions of $I=$ $\left\{i_{1}, \ldots, i_{m}\right\}$ into $k$ subsets $\left\{J_{1}, \ldots, J_{k}\right\}=g \in \mathcal{P}_{m: k}$ so that $\bigcup_{p=1}^{k} J_{p} \equiv I, J_{p} \cap J_{p^{\prime}}=\varnothing$ if $p \neq p^{\prime},\left|J_{p}\right|=\ell_{p}\left(\sum_{p=1}^{k} \ell_{p}=m\right)$. The CDF are:

$$
\begin{aligned}
& \partial_{J}^{m+m^{\prime}} c_{X}(\Delta \boldsymbol{p})=\frac{\partial^{m+m^{\prime}}}{\partial h_{i_{1}} \ldots \partial h_{i_{m}} \partial h_{j_{1}} \ldots \partial h_{j_{m^{\prime}}}} c_{X}(\Delta \boldsymbol{p}) \\
& =\sum_{k=1}^{m} \frac{\partial^{k+m^{\prime}} c_{X}(\Delta \boldsymbol{p})}{\partial \Delta \boldsymbol{p}^{k} \partial h_{j_{1}} \ldots \partial h_{j_{m^{\prime}}}}\left[\sum_{g \in \mathcal{P} P_{h ; k}}\left(\prod_{p=1}^{k} \partial_{J_{p} p}^{\ell} \Delta \boldsymbol{p}\right)\right]
\end{aligned}
$$

where $c_{X}(\Delta \boldsymbol{p})$ denotes the space-time covariance of an attribute $X(\boldsymbol{p}), \boldsymbol{p}=(\boldsymbol{s}, t) \in R^{n} \times T$. For the special case in which $m$ ' $=0$, Equation $(5 \mathrm{a})$ reduces to:

$$
\begin{aligned}
& \partial_{J}^{m} c_{X}(\Delta \boldsymbol{p})=\frac{\partial^{m}}{\partial h_{j_{1}} \ldots \partial h_{j_{m}}} c_{X}(\Delta \boldsymbol{p})=\sum_{k=1}^{m} \frac{\partial^{k} c_{X}(\Delta \boldsymbol{p})}{\partial \Delta \boldsymbol{p}^{k}}\left[\Sigma_{g \in \mathcal{P} \mathbb{P}_{n ; k}}\right. \\
& \left.\left(\prod_{p=1}^{k} \partial_{J_{p} p}^{\ell} \Delta \boldsymbol{p}\right)\right]
\end{aligned}
$$

For each $k=1, \ldots, m$, the number of elements in the class $\boldsymbol{P}_{m: k}$ is the Stirling number of the $2^{\text {nd }}$-kind, $S(m, k)=\left\{\begin{array}{l}m \\ k\end{array}\right\}=\frac{1}{k !}$ $\sum_{j=0}^{k}(-1)^{k-j}\left(\begin{array}{c}k \\ j\end{array}\right) j^{m}$. Worth-noticing special cases of Equations $(5 a)-(5 b)$ are:

$\frac{\partial}{\partial \boldsymbol{h}} c_{X}(\Delta \boldsymbol{p})=\frac{\partial \Delta \boldsymbol{p}}{\partial \boldsymbol{h}} \frac{\partial c_{X}}{\partial \Delta \boldsymbol{p}}$

$\frac{\partial^{2}}{\partial \boldsymbol{h}^{T} \partial \boldsymbol{h}} c_{X}(\Delta \boldsymbol{p})=\frac{\partial^{2} \Delta \boldsymbol{p}}{\partial \boldsymbol{h}^{T} \partial \boldsymbol{h}} \frac{\partial c_{X}}{\partial \Delta \boldsymbol{p}}+\left(\frac{\partial \Delta \boldsymbol{p}}{\partial \boldsymbol{h}}\right)^{T}\left(\frac{\partial \Delta \boldsymbol{p}}{\partial \boldsymbol{h}}\right) \frac{\partial^{2} c_{X}}{\partial \Delta \mathbf{p}^{2}}$

An apparent feature of CDF is that they decompose the covariance derivatives with respect to space $\left(h_{1} \ldots h_{n}\right)$ and time $\left(h_{0}=\tau\right)$ lags, commonly encountered in physical covariance 
laws, in terms of the metric $(\Delta p)$ and covariance derivatives. By combining MDF with CDF we find expressions explicitly containing the metric coefficients:

$\frac{\partial c_{X}}{\partial \boldsymbol{h}}=\left(\boldsymbol{h}^{T} \boldsymbol{E}+\frac{1}{2} \boldsymbol{\varepsilon}^{T}\right) \Delta \boldsymbol{p}^{-1} \frac{\partial c_{X}}{\partial \Delta \boldsymbol{p}}$

$\frac{\partial^{2} c_{X}}{\partial \boldsymbol{h}^{T} \partial \boldsymbol{h}}=\left[\Delta \boldsymbol{p}^{-1} \boldsymbol{E}-\left(\boldsymbol{E} \boldsymbol{h}+\frac{1}{2} \boldsymbol{\varepsilon}\right)\left(\boldsymbol{h}^{T} \boldsymbol{E}+\frac{1}{2} \boldsymbol{\varepsilon}^{T}\right) \Delta \boldsymbol{p}^{-3}\right] \frac{\partial c_{X}}{\partial \Delta \boldsymbol{p}}+$

$\left(\boldsymbol{E} \boldsymbol{h}+\frac{1}{2} \boldsymbol{\varepsilon}\right)\left(\boldsymbol{h}^{T} \boldsymbol{E}+\frac{1}{2} \boldsymbol{\varepsilon}^{T}\right) \Delta \boldsymbol{p}^{-2} \frac{\partial^{2} c_{X}}{\partial \Delta \boldsymbol{p}^{2}}$ space $\times$ time covariance-metric ratio $(\mathrm{CMR})$ :

$$
\partial_{J / J^{\prime}}^{\left(\boldsymbol{\kappa} / \boldsymbol{\kappa}^{\prime}\right)}=\partial_{j_{0}, \ldots, j_{\ell} / j_{0}^{\prime}, \ldots, j_{m}^{\prime}}^{\left(\kappa_{0}, \ldots, \kappa_{n} / \kappa^{\prime}, \ldots, \kappa_{n}^{\prime}\right)}=\frac{\frac{\partial^{\kappa}}{\partial h_{j_{0}}^{\kappa_{0}} \ldots \partial h_{j_{\ell}}^{\kappa_{\ell}}} c_{X}(\Delta \boldsymbol{p})}{\frac{\partial \kappa^{\prime}}{\partial h_{j j_{0}^{\prime}}^{\kappa_{0}^{\prime}} \ldots \partial h_{j_{m}^{\prime}}^{\kappa_{m}^{\prime}}} c_{X}(\Delta \boldsymbol{p})}
$$

where $\kappa_{p}, \kappa_{q}^{\prime}(p=0, \ldots, \ell ; q=0, \ldots, m ; \ell \leq m)$ are positive integers representing the order of differentiation, and $\kappa=\sum_{i=0}^{n} \kappa_{i}$, $\kappa^{\prime}=\sum_{i=0}^{n} \kappa_{i}^{\prime}$. The CMR are expressed geometrically in terms of the space-time metric (CMR determined in terms of $\Delta p$-derivative ratios).

We focus on up to $2^{\text {nd }}$-order space $\times$ time CMR that are special cases of the general CMR Equation (8), as follows:

$\partial_{i / 0}^{(1 / 1)}=\frac{\sum_{j=1}^{n} \varepsilon_{i j} h_{j}+\varepsilon_{0 i} \tau+\frac{1}{2} \varepsilon_{i}}{\sum_{j=1}^{n} \varepsilon_{0 j} h_{j}+\varepsilon_{00} \tau+\frac{1}{2} \varepsilon_{0}}\left(\right.$ for $\ell=m=\kappa_{i}=1(i \neq 0)$,

$\left.\kappa_{0}^{\prime}=1\right)$

$\partial_{i / j}^{(1 / 1)}=\frac{\sum_{j=1}^{n} \varepsilon_{i j} h_{j}+\varepsilon_{0 i} \tau+\frac{1}{2} \varepsilon_{i}}{\sum_{i=1}^{n} \varepsilon_{i j} h_{i}+\varepsilon_{0 j} \tau+\frac{1}{2} \varepsilon_{j}}\left(\right.$ for $\ell=m=\kappa_{i}=\kappa_{j}^{\prime}=1(i$,

$j \neq 0))$

$\partial_{i / 0}^{(2 / 1)}=\frac{\partial}{\partial h_{i}}\left(\partial_{i / 0}^{(1 / 1)}\right)+\zeta_{i} \partial_{i / 0}^{(1 / 1)}\left(\right.$ for $\ell=2, m=1, \kappa_{i}=2(i \neq 0)$,

$\left.\kappa_{0}^{\prime}=1\right)$

$\partial_{i, j / 0}^{(1,1 / 1)}=\frac{\partial}{\partial h_{j}}\left(\partial_{i / 0}^{(1 / 1)}\right)+\zeta_{j} \partial_{i / 0}^{(1 / 1)}\left(\ell=2, m=\kappa_{i}=1(i \neq 0), \kappa_{j}=1\right.$

$\left.(j \neq 0, j \neq i), \kappa_{0}^{\prime}=1\right)$

The $1^{\text {st }}$-order CMR are expressed in terms of the metric coefficients $\varepsilon_{i}, \varepsilon_{i j}$, whereas the $2^{\text {nd }}$-order CMR as functions of the $1^{\text {st }}$-order CMR and the derived metric coefficients $\zeta_{i}$. Since, together with $\varepsilon_{i}$ and $\varepsilon_{i j}$, the $\zeta_{i}$ are considered the unknown variables to be determined by the proposed approach (Section 2.3), a more detailed description of their internal structure and expli- $\varepsilon_{00}(\boldsymbol{h})=\sum_{i=1}^{n} \alpha_{00 ; i} h_{i}$

$\varepsilon_{i}(\tau)=\alpha_{i ; 0} \tau(i \neq 0)$

$\varepsilon_{0}(\boldsymbol{h})=\sum_{i=1}^{n} \alpha_{0 ; i} h_{i}\left(\alpha_{i j ; 0}=\alpha_{j i ; 0} ; i, j=0, . ., n\right)$

cit formulation is postponed until Section 3.2 later. For illusation, consider the metric of Equation (1) with metric coeffi$\times T$, i.e.:

$\varepsilon_{i j}(\tau)=\alpha_{i j ; 0} \tau(i, j \neq 0)$

$\varepsilon_{0 i}(\boldsymbol{h}, \tau)=\sum_{j=1}^{n} \alpha_{0 i ; j} h_{j}+\alpha_{0 i ; 0} \tau(i \neq 0)$

The MDF are as follows:

$$
\begin{aligned}
& \frac{\partial \Delta \boldsymbol{p}}{\partial h_{i}}=\frac{1}{2}\left[\tau\left(2 \sum_{j=1}^{n}\left(\alpha_{i j ; 0}+\alpha_{0 i ; j}+\alpha_{0 j ; i}\right) h_{j}+\left(\alpha_{i ; 0}+\alpha_{0 ; i}\right)\right)+\right. \\
& \left.\tau^{2}\left(2 \alpha_{0 i ; 0}+\alpha_{00 ; i}\right)\right] \Delta \boldsymbol{p}^{-1} \\
& \frac{\partial^{2} \Delta \boldsymbol{p}}{\partial h_{i}^{2}}=\left[\alpha_{i i} \tau-\left(\frac{\partial \Delta \boldsymbol{p}}{\partial h_{i}}\right)^{2}\right] \Delta \boldsymbol{p}^{-1} \\
& \frac{\partial \Delta \boldsymbol{p}}{\partial \tau}=\frac{1}{2}\left\{\sum_{i, j=1}^{n}\left(\alpha_{i j ; 0}+\alpha_{0 i ; j}+\alpha_{0 j ; i}\right) h_{i} h_{j}+\sum_{i=1}^{n}\left[\left(\alpha_{i ; 0}+\alpha_{0 ; i}\right) h_{i}\right.\right. \\
& \left.\left.+2\left(2 \alpha_{0 i ; 0}+\alpha_{00 ; i}\right) h_{i} \tau\right]\right\} \Delta \boldsymbol{p}^{-1} \\
& \frac{\partial^{2} \Delta \boldsymbol{p}}{\partial h_{i}^{2}}=\left[\alpha_{i i} \tau-\left(\frac{\partial \Delta \boldsymbol{p}}{\partial h_{i}}\right)^{2}\right] \Delta \boldsymbol{p}^{-1} \\
& \frac{\partial^{2} \Delta \boldsymbol{p}}{\partial h_{i} \partial \tau}=\left[\sum_{j=1}^{n}\left(\alpha_{i j ; 0}+\alpha_{0 i ; j}+\alpha_{0 j ; i}\right) h_{j}+\frac{1}{2}\left(\alpha_{i ; 0}+\alpha_{0 ; i}\right)+\tau\left(2 \alpha_{0 i ; 0}\right.\right. \\
& \left.\left.+\alpha_{00 ; i}\right)-\frac{\partial \Delta \boldsymbol{p}}{\partial h_{i}} \frac{\partial \Delta \boldsymbol{p}}{\partial \tau}\right] \Delta \boldsymbol{p}^{-1} \\
& \frac{\partial^{2} \Delta \boldsymbol{p}}{\partial h_{i} \partial h_{j}}=\left[\left(\alpha_{i j ; 0}+\alpha_{0 i ; j}+\alpha_{0 j ; i}\right) \tau-\frac{\partial \Delta \boldsymbol{p}}{\partial h_{i}} \frac{\partial \Delta \boldsymbol{p}}{\partial h_{j}}\right] \Delta \boldsymbol{p}^{-1}
\end{aligned}
$$

with the corresponding CMR:

$$
\begin{aligned}
& \partial_{i / 0}^{(1 / 1)}=\left[2 \sum_{j=1}^{n}\left(\alpha_{i j ; 0}+\alpha_{0 i ; j}+\alpha_{0 j ; i}\right) h_{j} \tau+\left(2 \alpha_{0 i ; 0}+\alpha_{00 ; i}\right) \tau^{2}+\right. \\
& \left.\left(\alpha_{i ; 0}+\alpha_{0 ; i}\right) \tau\right] /\left[\sum_{k, j=1}^{n}\left(\alpha_{k j ; 0}+\alpha_{0 k ; j}+\alpha_{0 j ; k}\right) h_{k} h_{j}+\sum_{j=1}^{n}\left(\alpha_{j ; 0}+\right.\right. \\
& \left.\left.\alpha_{0 ; j}\right) h_{j}+2 \sum_{j=1}^{n}\left(2 \alpha_{0 j ; 0}+\alpha_{00 ; j}\right) h_{j} \tau\right]
\end{aligned}
$$


$\partial_{i / j}^{(1 / 1)}=\left[2 \sum_{k=1}^{n}\left(\alpha_{i k ; 0}+\alpha_{0 i ; k}+\alpha_{0 k ; i}\right) h_{k}+\left(2 \alpha_{0 i ; 0}+\alpha_{00 ; i}\right) \tau+\alpha_{i ; 0}\right.$

$\left.+\alpha_{0 ; i}\right] /\left[2 \sum_{\ell=1}^{n} \alpha_{j \ell}\left(\alpha_{j \ell ; 0}+\alpha_{0 j ; \ell}+\alpha_{0 \ell ; j}\right) h_{\ell}+\left(2 \alpha_{0 j ; 0}+\alpha_{00 ; j}\right) \tau\right.$

$\left.+\alpha_{j ; 0}+\alpha_{0 ; j}\right]$

$\partial_{i / 0}^{(2 / 1)}=\frac{\partial}{\partial h_{i}}\left(\partial_{i / 0}^{(1 / 1)}\right)+\zeta_{i} \partial_{i / 0}^{(1 / 1)}$

$\partial_{i, j / 0}^{(1,1 / 1)}=\frac{\partial}{\partial h_{j}}\left(\partial_{i / 0}^{(1 / 1)}\right)+\zeta_{j} \partial_{i / 0}^{(1 / 1)}$

Joint "metric-covariance" partial differential equations (PDE) are obtained from the CMR above. For example, a direct result of the CMR Equations (9a) and (9b) are the PDE:

$\nabla \Delta \boldsymbol{p} \frac{\partial c_{X}}{\partial \tau}-\frac{\partial \Delta \boldsymbol{p}}{\partial \tau} \nabla c_{X}=0$

$\nabla \Delta \boldsymbol{p}\left(\nabla c_{X}\right)^{T}-\nabla c_{X}(\nabla \Delta p)^{T}=0$

where:

$\nabla \Delta \boldsymbol{p}=\left[\frac{\partial \Delta \boldsymbol{p}}{\partial h_{1}} \ldots \frac{\partial \Delta \boldsymbol{p}}{\partial h_{n}}\right]^{T}$

$\nabla c_{X}=\left[\frac{\partial c_{X}}{\partial h_{1}} \ldots \frac{\partial c_{X}}{\partial h_{n}}\right]^{T}$

For illustration, the components of Equations (13a) and (13b) are, respectively, $\left(\partial \Delta \boldsymbol{p} / \partial h_{i}\right)\left(\partial c_{X} / \partial \tau\right)-(\partial \Delta \boldsymbol{p} / \partial \tau)\left(\partial c_{X} / \partial h_{i}\right)=0(i=$ $1, \ldots, n)$, and $\left(\partial \Delta \boldsymbol{p} / \partial h_{i}\right)\left(\partial c_{X} / \partial h_{i}\right)-\left(\partial \Delta \boldsymbol{p} / \partial h_{i}\right)\left(\partial c_{X} / \partial h_{i}{ }^{\prime}\right)=0\left(i, i^{\prime}\right.$ $=1, \ldots, n)$. A key feature of Eqs (13a-b) is that they explicitly link environmental (covariance) changes with geometrical (metric) changes in space and time. A covariance with the general metric of Equation (1) must satisfy Equations (13a) - (13b), which is why these equations play a central role in the determination of a physically meaningful space-time metric.

Because of its special physical features, particularly interesting is the case of the traveling metric of Equation $(3 \mathrm{~g})$ with coefficients $\varepsilon_{0 i}$ being themselves functions of space-time. The following PDE representations of the space-time covariance are obtained from the CMR of Equations (9a) and (9d):

$\frac{\partial c_{X}}{\partial \tau}-\boldsymbol{\theta}^{T} \nabla c_{X}=0$

$\nabla \frac{\partial c_{X}}{\partial \tau}-\boldsymbol{\Theta}^{T} \nabla c_{X}-\boldsymbol{H}_{c} \boldsymbol{\theta}=0$

where

$\boldsymbol{\theta}=\left[\theta_{1} \ldots \theta_{n}\right]^{T}$

$\nabla c_{X}=\left[\frac{\partial c_{X}}{\partial h_{1}} \cdots \frac{\partial c_{X}}{\partial h_{n}}\right]^{T}$

$$
\begin{aligned}
& \nabla \frac{\partial c_{X}}{\partial \tau}=\left[\frac{\partial^{2} c_{X}}{\partial h_{1} \partial \tau} \cdots \frac{\partial^{2} c_{X}}{\partial h_{n} \partial \tau}\right]^{T} \\
& \boldsymbol{\Theta}=\left[\begin{array}{c}
\frac{\partial \theta_{1}}{\partial h_{1}} \frac{\partial \theta_{1}}{\partial h_{2}} \ldots \frac{\partial \theta_{1}}{\partial h_{n}} \\
\vdots \\
\frac{\partial \theta_{n}}{\partial h_{1}} \frac{\partial \theta_{n}}{\partial h_{2}} \ldots \frac{\partial \theta_{n}}{\partial h_{n}}
\end{array}\right] \\
& \mathbf{H}_{c}=\left[\begin{array}{c}
\frac{\partial^{2} c_{X}}{\partial h_{1}^{2}} \frac{\partial^{2} c_{X}}{\partial h_{1} \partial h_{2}} \ldots \frac{\partial^{2} c_{X}}{\partial h_{1} \partial h_{n}} \\
\vdots \\
\frac{\partial^{2} c_{X}}{\partial h_{n} \partial h_{1}} \frac{\partial^{2} c_{X}}{\partial h_{n} \partial h_{2}} \ldots \frac{\partial^{2} c_{X}}{\partial h_{n}^{2}}
\end{array}\right]
\end{aligned}
$$

The $\boldsymbol{H}_{c}$ is the Hessian matrix, and the vector $\boldsymbol{\theta}$ denotes the traveling vector function of the field, with directional components:

$\theta_{i}=\frac{\varepsilon_{0 i}+\tau \frac{\partial \varepsilon_{0 i}}{\partial \tau}}{1-\tau \frac{\partial \varepsilon_{0 i}}{\partial h_{i}}}$

in the $(\boldsymbol{h}, \tau)$-domain. Interpretation of the above PDE in the context of the real-world situation of interest offers valuable insight into environmental attribute distribution across spacetime. Generally, these PDE express quantitatively the relationship between the rates of covariance changes that reflect the corresponding changes of space-time dependence between attribute values at different points. In Equation (15a) the temporal rate of covariance change is explained as the spatial rate of covariance change multiplied by the corresponding traveling vector $\boldsymbol{\theta}$. Equation (15b) involves $\boldsymbol{\theta}$-derivatives requiring consistency between neighboring attribute values. Another implication of the PDE representations of space-time attribute dependence is that a traveling covariance function must satisfy Equations (15a-b), which is why these PDE can play a key role in the determination of a physically meaningful metric.

Each $\theta_{i}$ is a function of $\varepsilon_{0 i}$ along direction $s_{i}$, its spatial and temporal rates of change, and the lags considered. In the special case that the coefficients $\varepsilon_{0 i}$ are space- and time-independent, $\theta_{i}=\varepsilon_{0 i}$, and Equations (15a-b) reduce to:

$\frac{\partial c_{X}}{\partial \tau}-\varepsilon_{0}^{T} \nabla c_{X}=0$

$\nabla \frac{\partial c_{X}}{\partial \tau}-\boldsymbol{\varepsilon}_{0}^{T} \boldsymbol{H}_{c}=0$

where

$\boldsymbol{\varepsilon}_{0}=\left[\varepsilon_{01} \ldots \varepsilon_{0 n}\right]^{T}$

A covariance with the traveling space-time metric (3g) 
must satisfy Equations (17a) - (17b) in the general case of space- and time-dependent $\varepsilon 0$, or Equations (17a) - (17b) in the special case of space- and time-independent $\varepsilon_{0 i}$. Furthermore, for space- and time-independent $\varepsilon_{0 i}$ the following condition applies:

$\frac{\partial c_{X}}{\partial \tau}-\left(\boldsymbol{H}_{c}^{-1} \nabla \frac{\partial c_{X}}{\partial \tau}\right)^{T} \nabla c_{X}=0$

i.e., the covariance must satisfy Equation (19) in the case of space- and time-independent velocity.

\subsection{A Space-Time Metric Determination Approach}

The above analysis leads to a three-step approach for determining a space-time metric. The starting point is the equation governing the covariance $c_{X}(\Delta p)$ of the attribute of interest $X(p)$, since the covariance is a function of the space-time metric to be determined.

Step 1: Assume that the equation satisfied by the covariance $c_{X}(\Delta \boldsymbol{p})$ of the environmental attribute $X(\boldsymbol{p})$-considered as a random field in the $n$-dimensional space $\times$ time $\left(R^{n} \times T\right)$ domain with $\boldsymbol{p}=(\boldsymbol{s}, t)=\left(s_{1}, \ldots, s_{n}, t\right)$ - is:

$L_{c}\left[c_{X}, \boldsymbol{a}\right]=0$

where $L_{c}$ denotes "law of $c_{X}$ ", and $\boldsymbol{a}=\left\{a_{k}\right\}(k=1, \ldots, \eta)$ is a set of coefficients (determined by means of the stochastic expectation process leading from the physical law governing $X(\boldsymbol{p})$ to the $L_{c}$-equation above; see literature on stochastic differential equations and their environmental applications (e.g., Srinivasan and Vasudevan, 1971; Soong, 1973; Dobrovolski, 2010; Klyatskin, 2015).

Step 2: Using the CMR expressions introduced in the previous sections, the $L_{c}$-Equation (20) can be rewritten as:

$$
L_{G}\left[\partial_{J / J^{\prime}}^{\left(\boldsymbol{\kappa} / \boldsymbol{\kappa}^{\prime}\right)}, \boldsymbol{a}\right]=0
$$

where $\partial_{J / J^{\prime}}^{\left(\boldsymbol{\kappa} / \boldsymbol{\kappa}^{\prime}\right)}$ were defined earlier in terms of the metric coefficients $(\boldsymbol{E}, \boldsymbol{\varepsilon})$. I.e., the physical equation $\left(L_{c}\right)$ has been replaced by the geometrical equation $\left(L_{G}\right)$ that the space-time metric coefficients must satisfy.

Step 3: Solve Equation (25) with respect to the metric coefficients $(\boldsymbol{E}, \boldsymbol{\varepsilon})$ expressed in terms of the physical law parameters $\boldsymbol{a}$, viz.:

$$
\{\boldsymbol{E}, \boldsymbol{\varepsilon}\}=\{\boldsymbol{E}(\boldsymbol{a}), \boldsymbol{\varepsilon}(\boldsymbol{a})\}=\left\{\varepsilon_{00}\left(a_{k}\right), \varepsilon_{0 i}\left(a_{k}\right), \varepsilon_{i j}\left(a_{k}\right), \varepsilon_{i}\left(a_{k}\right), \varepsilon_{0}\left(a_{k}\right)\right\}
$$

$(i, j=1, \ldots, n ; k=1, \ldots, \eta)$. There may exist more than one solution, leading to different metrics $\Delta \boldsymbol{p}$ (revealing different space-time dependence structures). One may also find that certain solutions recur in various disguises.

A few more comments can be made concerning the metric determination approach. Sources of uncertainty maybe the boundary/initial conditions (BIC) or the coefficients of the physical law governing the environmental attribute $X(p)$ of interest. The corresponding BIC of the $c_{X}$ should be included in the $L_{G}$ formulation above (see examples is Section 5). One cannot specify the metric coefficients and the attribute characteristics (uncertainty sources, BIC) independently, since they are connected via the geometrical equation $L_{G}$.

Formally, any metric solution is acceptable if it satisfies the $L_{G}$-equations above. In this sense, two main types of solutions can be considered: exact solutions that are based on certain assumptions (concerning metric symmetry or the mathematical covariance structure), and approximate (analytical and numerical) solutions that are able to explore different environmental situations. The solutions generally lead to a set of dependent equations (in the sense that the space-time metric coefficients usually appear in more than one equation). Having a general metric form, such as Equations (1) and (2), one can make assumptions on those components of the physical law for which the corresponding terms in the $L_{G}$-equation are not automatically determined, and then solve the remaining equations. Concerning their usefulness, exact solutions (though often obtained by imposing simplifying assumptions) are valuable in certain ways: they allow a deeper understanding of the methodological underpinnings of the metric determination approach, all quantities are expressed by elementary functions or well-known special functions, and they can complement the approximate solutions either by providing the background on which approximations for real environmental situations can be built or by enabling numerical accuracy checks.

\section{Results}

\subsection{Applications}

As already noted, the physical covariance equations $\left(L_{C}\right.$ in Step 1 above) are assumed known (their derivation from the original environmental attribute law is not the concern of the metric determination approach, since it can be found in the relevant stochastic environmental modeling literature). Accordingly, metric-solutions are obtained based on certain assumptions: the original physical laws are expressed in terms of stochastic PDE (in which the uncertainty sources are either random law BIC or random law coefficients), the corresponding $L_{C^{-}}$ equations are derived from the physical laws by means of stochastic expectation, and more than one space-time solution of the $L_{G}$-equation may be possible, admitting different interpretations.

In Example 1 (Table 2) the $L_{C}$-equation in the $R^{3} \times T$ domain is given by Equation (23), where $a(<0)$ is a physical coefficient, and $\nabla=\sum_{i=1}^{3} \partial / \partial h_{i}$. Equation (23) is the covariance equation encountered in three-dimensional space $\times$ time statistical turbulence studies (Monin and Yaglom, 1971). Under certain conditions, the corresponding attribute $X(\boldsymbol{p})$ is called a "frozen field" (Taylor, 1938), i.e., it may be seen as a field that "travels" along a specified direction with a certain velocity. In theory, this $L_{C}$-equation can be associated with the traveling metric of Equation (3g). Equation (23) can be re-written in the $L_{G}$-form of Equation (24), where the $\mathrm{CMR} \partial_{i / 0}^{(1 / 1)}$ is given by 
Table 2. Space-time Metric Determination Examples

\begin{tabular}{|c|c|c|c|c|c|c|}
\hline & Example $1\left(R^{3} \times T\right)$ & Example $2\left(R^{3} \times T\right)$ & Example $3\left(R^{2} \times T\right)$ & & Example $4\left(R^{1} \times T\right)$ & \\
\hline$L_{C}$ & $\left(\frac{\partial}{\partial \tau}+a \sum_{i=1}^{3} \frac{\partial}{\partial h_{i}}\right) c_{X}=0$ & $\sum_{i=1}^{3}\left(h_{i} \frac{\partial}{\partial \tau}-a^{2} \tau \frac{\partial}{\partial h_{i}}\right) c_{X}=0$ & {$\left[\xi \frac{\partial}{\partial \tau}-\mathbf{k} \cdot \nabla-\kappa \nabla^{2}\right] c_{X}=0$} & (37) & $\left.\begin{array}{l}{\left[S \frac{\partial}{\partial \tau}-K \frac{\partial^{2}}{\partial h^{2}}\right] c_{X}=0} \\
c_{X}(h, 0)=c_{0} e^{-3 h / a_{r}} \\
c_{X}(0, \tau)=c_{0} e^{3 \tau / a_{\tau}} \\
c_{X}(L, \tau)=0\end{array}\right\}$ & (46) \\
\hline$\overline{L_{G}}$ & $\begin{array}{l}\frac{1}{a}+\sum_{i=1}^{3} \partial_{i / 0}^{(1 / 1)}=0 \\
\sum_{i=1}^{3}\left(\sum_{j=1}^{3} \varepsilon_{j i} h_{j}+\varepsilon_{0 i} \tau+\frac{1}{2} \varepsilon_{i}\right) \\
+\frac{1}{a}\left(\sum_{i=1}^{3} \varepsilon_{0 i} h_{i}+\varepsilon_{00} \tau+\frac{1}{2} \varepsilon_{0}\right) \\
=0\end{array}$ & $\begin{array}{l}\sum_{i=1}^{3}\left(\partial_{i / 0}^{(1 / 1)}-\frac{1}{a^{2} \tau} h_{i}\right)=0 \\
\sum_{i=1}^{3}\left(\sum_{j=1}^{3} \varepsilon_{j i} h_{j}+\varepsilon_{0 i} \tau+\frac{1}{2} \varepsilon_{i}\right)- \\
\frac{1}{a^{2} \tau}\left(\sum_{i=1}^{3} \varepsilon_{0 i} h_{i}+\varepsilon_{00} \tau+\frac{1}{2} \varepsilon_{0}\right) \sum_{i=1}^{3} h_{i} \\
=0\end{array}$ & 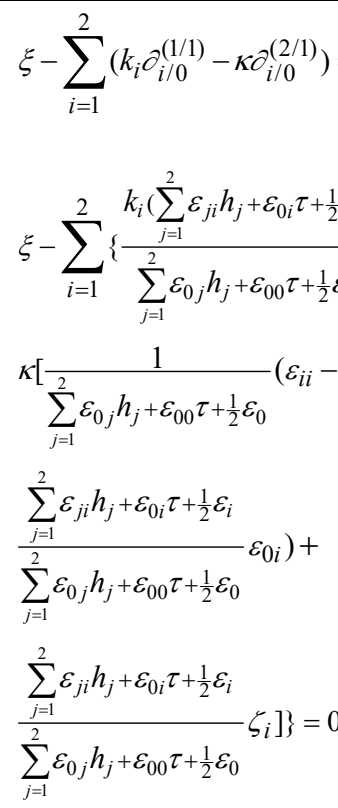 & $0 \quad$ (39) & $\begin{array}{l}\frac{S}{K}-\partial_{1 / 0}^{(2 / 1)}=0 \\
\frac{S}{K}-\frac{1}{\varepsilon_{01} h+\varepsilon_{00} \tau+\frac{1}{2} \varepsilon_{0}}\left(\varepsilon_{1}\right. \\
\left.\frac{\varepsilon_{11} h+\varepsilon_{01} \tau+\frac{1}{2} \varepsilon_{1}}{\varepsilon_{01} h+\varepsilon_{00} \tau+\frac{1}{2} \varepsilon_{0}} \varepsilon_{01}\right)- \\
\zeta_{1} \frac{\varepsilon_{11} h+\varepsilon_{01} \tau+\frac{1}{2} \varepsilon_{1}}{\varepsilon_{01} h+\varepsilon_{00} \tau+\frac{1}{2} \varepsilon_{0}}=0\end{array}$ & $\begin{array}{r}(48) \\
11- \\
(49)\end{array}$ \\
\hline$\varepsilon$ & $\left.\begin{array}{l}\varepsilon_{0}=\varepsilon_{i}=0 \\
\varepsilon_{j i}=1 \\
\varepsilon_{0 j}=-3 a \\
\varepsilon_{00}=9 a^{2}\end{array}\right\}$ & $\left.\begin{array}{l}\varepsilon_{0}=\varepsilon_{i}=\varepsilon_{0 i}=\varepsilon_{j i}=0 \\
\varepsilon_{i i}=1 \\
\varepsilon_{00}=a^{2} \\
\varepsilon_{0}=\varepsilon_{i}=\varepsilon_{0 i}=0 \\
\varepsilon_{1 j}=\varepsilon_{2 j}=\varepsilon_{3 j}=1 \\
\varepsilon_{00}=3 a^{2}\end{array}\right\}$ & $\left.\begin{array}{l}\varepsilon_{i j}=\varepsilon_{00}=\varepsilon_{0 i}=0 \\
\varepsilon_{0}=\xi^{-1} \sum_{i=1}^{2}\left(k_{i} \varepsilon_{i}-\kappa \varepsilon_{i}^{2}\right) \\
\zeta_{i}=-\varepsilon_{i} \\
\varepsilon_{i j}=\varepsilon_{00}=\varepsilon_{0 i}=0 \\
\varepsilon_{i}=-\zeta_{i}=1 \\
\varepsilon_{0}=\xi^{-1}\left(\sum^{2} k_{i}-2 \kappa\right)\end{array}\right\}$ & (40d-f) & $\left.\begin{array}{l}\varepsilon_{11}=\varepsilon_{00}=\varepsilon_{01}=0 \\
\varepsilon_{0}=-\frac{K}{S}\left(\frac{3}{a_{r}}\right)^{2} \\
\zeta_{1}=-\varepsilon_{1}=-\frac{3}{a_{r}}\end{array}\right\}$ & $(50 \mathrm{a}-\mathrm{c})$ \\
\hline$\Delta p^{2}$ & $\left(\sum_{i=1}^{3} h_{i}-3 a \tau\right)^{2}$ & $\begin{array}{l}\sum_{i=1}^{3} h_{i}^{2}+a^{2} \tau^{2} \\
\sum_{i=1}^{3} h_{i} h_{j}+3 a^{2} \tau^{2}\end{array}$ & $\sum_{i=1}^{2} h_{i}+\xi^{-1}\left(\sum_{i=1}^{2} k_{i}-2 \kappa\right) \tau$ & (41a) & $\begin{array}{l}\frac{3}{a_{r}} h-\left(\frac{3}{a_{r}}\right)^{2} \frac{K}{S} \tau \\
\left(h-\frac{K}{S} \tau\right)^{2}\end{array}$ & (51a) \\
\hline
\end{tabular}

Equation (9a). While $L_{c}$-Equation (23) describes the physical correlation pattern of the attribute $X(\boldsymbol{p}), L_{G}$-Equation (24) describes the geometrical pattern of the space-time domain within which $X(p)$ varies. In this sense, the metric $\Delta p$ itself may be seen as the "attribute" of the $L_{G}$-equation. By inserting Equation (9a) into Equation (24), the $L_{G}$-equation becomes Equation (25). The last equation is an analytical geometrical equation that replaces the physical covariance Equation (23), which 
means that the space-time metric coefficients must be chosen so that Equation (25) is satisfied. An obvious solution is given in Equations (26a-d). The space-time metric consistent with the $L_{G}$-equation is that of Equation (27), i.e., the traveling metric of Equation $(3 \mathrm{~g})$, as expected, in which case the covariance model representing the traveling random field is:

$c_{x}(\Delta \boldsymbol{p})=c_{x}(\boldsymbol{h}-\boldsymbol{a} \tau)=e^{-\left[\sum_{i=1}^{3}\left(h_{i}-a \tau\right)\right]^{2}}$

(which, indeed, satisfies Equation (23)). A metric solution implies relationships between the metric coefficients and the physical parameter $a$ that admit a certain interpretation. Specifically, the metric (27) is characterized by the positive $a$-effect on the correlation strength (range) between points, an effect that decreases with increasing time interval (this is a result of the covariance variation as a function of the $a$-dependent metric $\Delta p)$. This is, indeed, the case here, since the derived metric, Equation (27), is consistent with the frozen attribute field of the underlying physics.

Example 2 (Table 2) considers the covariance Equation (29) in $R^{3} \times T$, where $\boldsymbol{h}=\left(h_{1} h_{2} h_{3}\right)^{T}$, and $a$ is a constant (these equations are found in atmospheric environment situations, Daley, 1999). Equation (29) yields the $L_{G}$-Equation (30), where $\partial_{i / 0}^{(1 / 1)}$ is given by Equation (9a). By inserting Equation (9a) into (30), the latter becomes Equation (31). This $L_{G}$-equation, which represents an interaction of geometrical requirements combined with physical parameters, has more than one metric solutions, in which case it is interesting to understand what qualitative features each solution may possess. An obvious solution is Equations (32a-c) with the corresponding metric (33a) and Gaussian covariance:

$c_{X}(\Delta \boldsymbol{p})=e^{-\left(\sum_{i=1}^{3} h_{i}^{2}+a^{2} \tau^{2}\right)}$

Another solution of the $L_{G}$-equation is Equations (32d-f) with metric Equation (33b). The fact that more than one space-time metrics satisfy the $L_{G}$-equation is a situation similar to that in which more than one metric has been found to satisfy the fieldequations of gravitation (e.g., Minkowsky, Schwarzschild, Friedmann, and Walker metric solutions; Stephani et al., 2003; Zhu et al., 2015). We also observe that:

$$
\Delta \boldsymbol{p}_{(33 b)}^{2}=\Delta \boldsymbol{p}_{(33 a)}^{2}+\sum_{i \neq j=1}^{3} h_{i} h_{j}+2 a^{2} \tau^{2}
$$

Different metrics may rest on different physical bases (e.g., different dependence structures across space-time). $\Delta \boldsymbol{p}_{(33 a)}$ allows a rather smooth attribute variation in space-time, whereas $\Delta \boldsymbol{p}_{(33 b)}$ implies a rougher variation and a higher negative effect of the $a$-parameter on the correlation strength between points (this negative effect increases with increasing time interval). Similar to Example 1, this is a result of the variation of the corresponding covariance shapes as functions of $\Delta \boldsymbol{p}_{(33 a)}$ and $\Delta \boldsymbol{p}_{(33 b)}$ (covariance with metric $\Delta \boldsymbol{p}_{(33 a)}$ has a smaller slope at origin than that with $\Delta \boldsymbol{p}_{(33 b)}$, implying a smoother attribute variation).
By inserting the Gaussian model:

$c_{X}(\Delta \boldsymbol{p})=e^{-\left(\sum_{i=1}^{2} b_{i} h_{i}^{2}+c \tau^{2}\right)}$

into $L_{C}$-Equation (29) one finds, $b_{i}=c / a^{2}=b$, i.e., the model coefficients $b_{i}, c$ are chosen so that this relationship holds (in which case, the Gaussian covariance is compatible with $L_{C}$ ). From the above relationship the Gaussian model yields:

$c_{X}(\Delta \boldsymbol{p})=\left[e^{-\Delta p_{(33 a)}}\right]^{b}$

i.e., the covariance of Equation (35b) is a generalization of Equation (34) that, in addition to the physical law parameter $a$, includes the Gaussian model parameter $b$.

Example 3 (Table 2) considers a hydrologic situation involving the variation of the hydraulic head governed by the subsurface flow law in the $R^{2} \times T$ domain with source of uncertainty the random groundwater flow law parameters (hydraulic storativity and conductivity; Dagan, 1989). In this domain, the metric Equation (1) reduces to:

$$
\begin{aligned}
& \Delta \boldsymbol{p}^{2}=\sum_{i=1}^{2} \varepsilon_{i i} h_{i}^{2}+2 \tau \sum_{i=1}^{2} \varepsilon_{0 i} h_{i}+\sum_{i=1}^{2} \varepsilon_{i} h_{i}+\left(\varepsilon_{12}+\varepsilon_{21}\right) h_{1} h_{2} \\
& +\varepsilon_{00} \tau^{2}+\varepsilon_{0} \tau,
\end{aligned}
$$

and the covariance function characterizing the hydraulic head's space-time variation is expressed by the $L_{C}-$ Equation (37), where $\xi$ is the hydraulic storage coefficient, $\kappa$ is the hydraulic conductivity and we define $\partial \kappa / \partial h_{i}=k_{i}$, and $\boldsymbol{k}=\left(k_{1} k_{2}\right)^{\mathrm{T}}$. Following the metric determination approach, the $L_{G}$-equation corresponding to $L_{G}$-Equation (37) is expressed as in Equation (38), which, in light of Equation (36), is written in the analytical form of Equation (39). Equation (39) must be solved with respect to the metric coefficients $\varepsilon_{00}, \varepsilon_{0 i}, \varepsilon_{i j}, \varepsilon_{i}, \varepsilon_{0}$ and $\zeta_{i}(i$, $j=1,2)$. A solution set is Equations (40a-c), in which case the metric Equation (36) reduces to (41a), and the $L_{C}$-Equation (37) has the covariance solution:

$c_{X}(\Delta \boldsymbol{p})=e^{-\sum_{i=1}^{2} \varepsilon_{i} h_{i}-\varepsilon_{0} \tau}$,

where $\varepsilon_{0}$ is given by Equation (40b). For illustration, one could chose a unit value for $\varepsilon_{i}$ and define the corresponding $\varepsilon_{0}$ value, see Equations (40d-f) in which the metric coefficients are now functions only of the parameters $\xi, \kappa, k_{i}(i=1,2)$ of the hydrologic covariance law. Then, the metric (41a) reduces to that of (41b). On the other hand, one can find covariance models with metrics different than (41a), which do not satisfy the covariance law (37).

Space-time plays a dual role in the $L_{G}$-equations, because it constitutes both the object and the context within which space-time dependence is defined. This kind of self-reference gives $L_{G}$-equations governing metric $\Delta \boldsymbol{p}$ certain characteristics that are different than those of the equations governing the environmental attribute $X(\boldsymbol{p})$. For example, normally we can 
formulate the BIC of the physical law by specifying the $X(\boldsymbol{p})$ values at a given space-boundary and time-instant, and then use the physical law to determine the space-time evolution of $X(p)$. In contrast, due to the inherent self-referential aspect of $\Delta p$, one is not free to specify arbitrary BIC, but only conditions that already satisfy self-consistency requirements imposed by the $L_{G}$-equations themselves.

Example 4 (Table 2) is a special case of Example 3, representing transient groundwater flow in the $R^{1} \times T$ domain with the random source now being the flow BIC. Specifically, in $R^{1}$ $\times T$ the general metric of Equation (1) simply reduces to:

$\Delta p^{2}=\varepsilon_{11} h^{2}+\varepsilon_{00} \tau^{2}+2 \varepsilon_{01} h \tau+\varepsilon_{1} h+\varepsilon_{0} \tau$

and the governing space $\times$ time physical law is (Zhang, 2002):

$\left[\frac{S}{K} \frac{\partial}{\partial t}-\frac{\partial^{2}}{\partial s^{2}}\right] X(s, t)=0$

Hydraulic head $X(s, t)$ is decomposed as $X(s, t)=\overline{X(s, t)}+X^{\prime}(s$, $t), \overline{X(s, t)}$ and $X^{\prime}(s, t)$ denote the head mean and head perturbation, respectively; $S$ is the storage coefficient; and $f(s)=$ $\log K(s)$, with constant saturated hydraulic conductivity $K$ across the study domain. The random BIC are:

$$
\begin{aligned}
& X(s, t=0)=H\left(1-\frac{s}{L}\right)+X^{\prime}(s, t=0) \\
& X(s=0, t)=H+X^{\prime}(s=0, t) \\
& X(s=L, t)=X^{\prime}(s=L, t)
\end{aligned}
$$

The hydraulic head covariance $L_{C}-$ equation associated with the groundwater flow law (44) is given by Equation (46), where $h$ $=s-s^{\prime}, \tau=t-t^{\prime}$, subject to the head covariance BIC (47a-c), where the study domain is much larger than the correlation length of head perturbations ( $L>>a_{r}$ ). The $L_{G}-$ equation corresponding to (46) is expressed by (48), which, in light of metric (43), takes the analytical form (49). Equation (49) must be solved with respect to the metric coefficients $\varepsilon_{00}, \varepsilon_{01}, \varepsilon 11, \varepsilon 1, \varepsilon 0$, $\zeta_{1}$. A solution set is given by Equations (50a-c) with space-time metric (51a).

It is interesting to consider the numerical solution $\hat{c}_{X}$ of the hydraulic head covariance $L_{C}-$ Equation (46), subject to the BIC (47a-c), and investigate if the numerical covariance $\hat{c}_{X}$ indeed admits a metric of the form (51a), i.e., it is of the theoretical covariance form:

$$
c_{X}(h, \tau)=c_{0} e^{-\frac{3}{a_{r}} h+\left(\frac{3}{a_{r}}\right)^{2} \frac{K}{S} \tau}
$$

For illustration, assume numerical values for the coefficients of the groundwater flow equation: $S=10^{-5} \mathrm{~m}, K(s)=0.135$ $\mathrm{m} /$ day, $a_{r}=10 \mathrm{~m}, a_{\tau}=0.2$ day, $L=200 \mathrm{~m}, T=2$ day, $c_{0}=10$ $\mathrm{m}^{2}$. The derived numerical $\hat{c}_{X}$ solution of $L_{C}-$ Equation (46) is plotted in Figure (1a). The perfect fit between $c_{X}$ and $\hat{c}_{X}$ (Fig- ure (1b)) implies that the numerical $\hat{c}_{X}$ can be expressed mathematically by the theoretical $c_{X}$ form of Equation (52) with metric (51a). Hence, for the groundwater flow law (44) with random BIC (45a) - (45c), the space-time metric (51a) is an appropriate choice. The coefficients $\varepsilon_{i}$ of the metric (41a) are not determined arbitrarily. It was found that these coefficients are functions of the spatial correlation length $a_{r}$ of the $L_{C}-$ BIC. The $\mathrm{BIC}(47 \mathrm{~b})$ shows that the temporal head covariance can increase exponentially with time. This is the case in practice, e.g., when the source of flow uncertainty is the random hydraulic conductivity coefficients. Nevertheless, in the present flow example the $c_{X}$ of Equation (52) does not address the regions of extreme high covariance values (large $\tau$ and small $h$ ), because the $c_{X}$-calculation requires that:

$h-\frac{K}{S} \tau \geq 0$.

This inequality has the physical meaning that the hydraulic head variations can only be affected by the noise of the upstream area due to either advection or diffusion mechanisms. Another solution set of $L_{G}$-Equation (49) is given by (50d-g), which corresponds to the metric (51b). This result should be expected if we observe that the $L_{C}-$ Equation (46) is of the traveling field form.

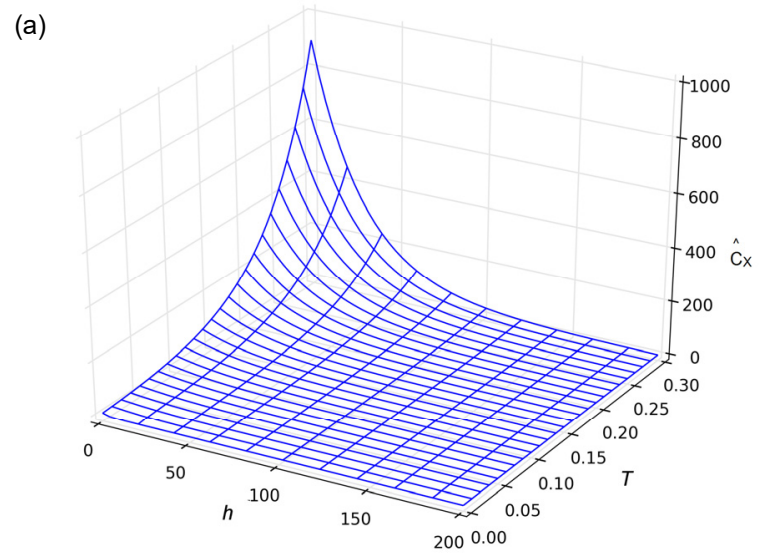

(b)

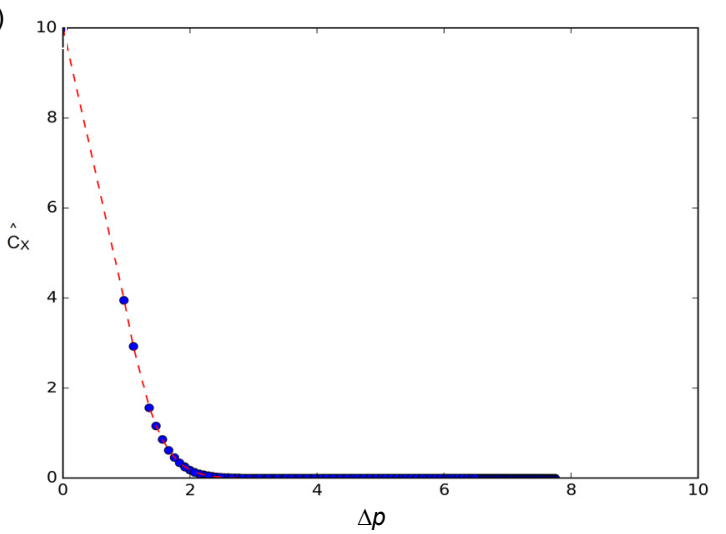

Figure 1. Plots of (a) numerical $\hat{c}_{X}$ solution (46); and (b) $\hat{c}_{X}$ solution (46) vs. $\Delta p$ of Equation (51a) (circled line), and theoretical $c_{X}$ model (52) vs. $\Delta p$ of (51a) (dashed line). 
Lastly, it is instructive to consider subsurface flow that is clearly inconsistent with the space-time metric (51a). For a non-constant hydraulic conductivity $K$, the $R^{1} \times T$ transient groundwater flow law governing the hydraulic head $X(s, t)$ is (Zhang, 2002):

$\left[\frac{S}{K(s)} \frac{\partial}{\partial t}-\frac{\partial f(s)}{\partial s} \frac{\partial}{\partial s}-\frac{\partial^{2}}{\partial s^{2}}\right] X(s, t)=0$

with BIC:

$$
\begin{aligned}
& X(s, t=0)=H\left(1-\frac{s}{L}\right) \\
& X(s=0, t)=H \\
& X(s=L, t)=0
\end{aligned}
$$

Unlike Equation (44), in this case the uncertainty source is the

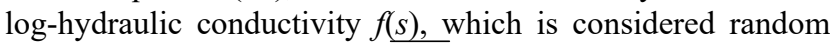
and is decomposed as $f(s)=\overline{f(s)}+f^{\prime}(s)$, where $f(s)=\log K(s)$ is the mean and $f^{\prime}(s)$ is the random fluctuation with variance $\sigma_{f}^{2}$. Using the stochastic perturbative-expansion method (Zhang, 2002), assuming $\partial \bar{f} / \partial s=0$, and a fixed location $\left(s^{\prime}, t^{\prime}\right)$ $=(0,0)$ so that $h=s-s^{\prime}=s, \tau=t-t^{\prime}=t$, the $L_{C}-$ equation of the flow law (54) is Equation (46), but with new BIC:

$c_{X}(h, 0)=0$

$c_{X}(0, \tau)=0$

$c_{X}(L, \tau)=0$

Equations (46) and (56a) - (56c) establish the space-time relationships between hydraulic head and conductivity with respect to the $1^{\text {st }}$-order hydraulic head or conductivity variation at a fixed location $\left(s^{\prime}, t^{\prime}\right)=(0,0)$. To obtain the numerical covariance solution of $L_{C}-$ Eq (46) subject to Eqs (56a-c), we assume that the covariance of the log-hydraulic conductivity is given by:

$c_{f}(h)=e^{-\frac{3 h}{a_{r}}}$

$H=50 \mathrm{~m}, S=10^{-5} \mathrm{~m}, K(s)=0.135 \mathrm{~m} /$ day $(\bar{f}=-2, \partial \bar{f} / \partial s=0)$, $c_{0}=0.23$, and $a_{r}=54 \mathrm{~m}$. The space and time domains are $L=$ $200 \mathrm{~m}$ and $T=2$ days, respectively, and the discretized space and time intervals are $\Delta s=4 \mathrm{~m}$ and $\Delta t=0.0025$ day, respectively. The resulting numerical head covariance $\hat{c}_{X}$ is plotted in Figure (2a). The temporal head covariance component of the plot in Figure (2a) can increase exponentially with time (which is consistent with Equation (47b)). Also, in Figure 2b we plot $\hat{c}_{X} v s$. the metric (51a) with $\varepsilon_{1}=1\left(a_{r}=3\right)$. The circles in this figure indicate that for a specified $\Delta p$-value there can be assigned several $\hat{c}_{X}$-values, corresponding to different combina- tions of $(h, \tau)$-values in (51a). This means that the covariance of the hydraulic head obeying Equation (54) subject to the BIC (55a) - (55c) cannot have a metric of the form (51a). In sum, the source of randomness (physical law BIC vs. law coefficients) can affect the form of the space-time metric: the metric (51a) is appropriate when the randomness in the physical law (44) is its $\mathrm{BIC}$, but it is inappropriate when the randomness source is the physical law coefficients.

\subsection{Concerning the Zeta Coefficients}

Any metric whatsoever is formally acceptable if it satisfies the $L_{G}$-equation, which means that, in a sense, this equation becomes a definition of the metric coefficients. In this context, a further interpretation of the zeta coefficients may be useful. Generally, these coefficients can be expressed as follows:

$\zeta_{i j}=\frac{\partial}{\partial h_{i}} \log \frac{\partial}{\partial h_{j}} c_{X}(\Delta p)$

$(i, j=0, \ldots, n)$, which also depends on the geometrical characteristics of the covariance. In the special case of interest in the earlier analysis $\left(j=0, h_{0}=\tau\right)$ :

$\zeta_{i 0}=\zeta_{i}=\frac{\partial}{\partial h_{i}} \log \frac{\partial}{\partial \tau} c_{X}(\Delta \boldsymbol{p})$

(a)

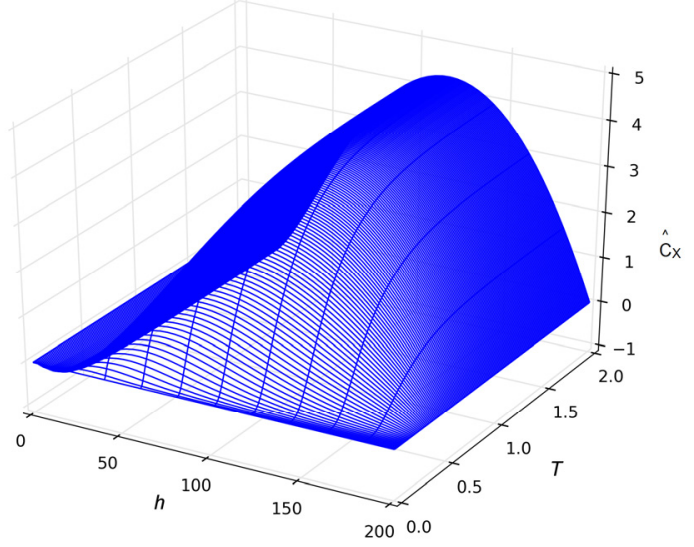

(b)

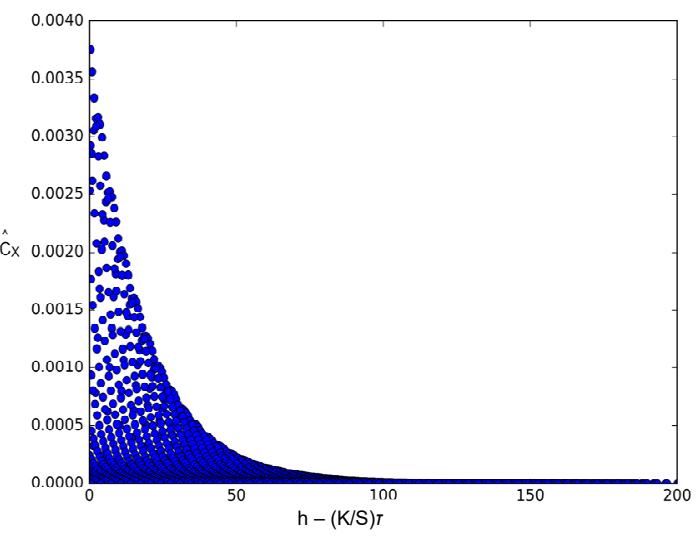

Figure 2. Plots of $(a) \hat{c}_{X}$ ( $1^{\text {st }}-$ order head) $v s$. $(h, \tau)$; and $(b) \hat{c}_{X}$ ( $1^{\text {st }}-$ order head $) v s . \Delta p=h-(K / S) \tau$. 


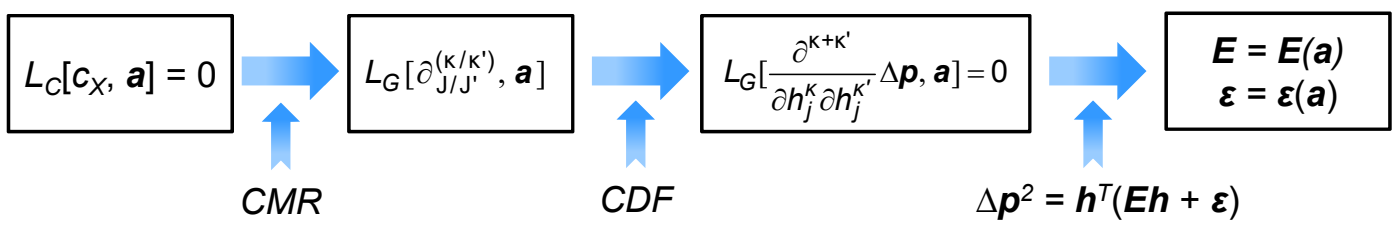

Figure 3. Space-time metric determination examples.

If information about the $c_{X}$ shape is available (or can be assumed), the $\zeta_{i j}$ or $\zeta_{i}$ can be calculated directly from Equations (58) - (59), in which case the zeta coefficient is expressed in terms of $\Delta \boldsymbol{p}$. As a matter of fact, the $\zeta_{i}$ can be calculated for several classes of covariance functions. For illustration, consider covariance functions belonging to the exponential-power class:

$\frac{\partial}{\partial \tau} c_{X}(\Delta \boldsymbol{p})=b\left\{\begin{array}{l}e^{\frac{\theta}{\rho+1} \Delta p^{\rho+1}} \quad(\rho \neq-1) \\ \Delta \boldsymbol{p}^{\eta+\theta}(\rho=-1)\end{array}\right.$

$(b, \theta, \rho \in R)$. In this case, we find that:

$\zeta_{i}=\theta \frac{\partial \Delta \boldsymbol{p}}{\partial h_{i}} \Delta \boldsymbol{p}^{\rho}$

i.e., $\zeta_{i}$ is expressed in terms of the $\Delta p$, as stated earlier. Then, the CMR equation $(9 \mathrm{c})$ becomes:

$\partial_{i / 0}^{(2 / 1)}=\frac{\partial}{\partial h_{i}}\left(\partial_{i / 0}^{(1 / 1)}\right)+\theta \frac{\partial \Delta \boldsymbol{p}}{\partial h_{i}} \Delta \boldsymbol{p}^{\rho} \partial_{i / 0}^{(1 / 1)}$

which links the space-time metric with the covariance parameters $\theta$ and $\rho$. Similar expressions can be derived for higherorder CMR.

It is interesting to revisit Example 3 (Table 2). As noted earlier, in the analysis of Example 3 the exact shape of model $c_{X}$ needed not be known in advance in order to determine the specific metric, Equation (41a). Instead, the coefficients $\varepsilon_{i}, \varepsilon_{i j}$ and $\zeta_{i}$ needed to be chosen so that they satisfy Equations (40a - c). In light of Equations (58) - (61) above, for the covariance solution $c_{X}$ of Equation (37) to be consistent with the metric solution of Eq (41a) it is sufficient that $c_{x}$ satisfies:

$\frac{\partial}{\partial h_{i}} \log \frac{\partial}{\partial \tau} c_{X}(\Delta p)=-\varepsilon_{i}$

Indeed, for the covariance of Equation (42), Equation (59) directly yields Equation (40c). The same result is obtained from Equations (61) and (36) by setting $\rho=1, \theta=-2$. Moreover, Equation (42) gives:

$\frac{\partial}{\partial \tau} c_{X}(\Delta p)=-\varepsilon_{0} c_{X}(\Delta p)$

which is the same result as that obtained from Equation (60a) for $b=-\varepsilon_{0}, \rho=1, \theta=-2$.

\section{Concluding Remarks}

Central among the quantitative features of a physical geometry is its metric structure, i.e., a set of mathematical expressions that define spatiotemporal distance (Christakos and Hristopulos, 1998; Curriero, 2006; Nieves et al., 2007). These expressions cannot always be defined unambiguously, but depend on two entirely different factors: a "relative" factor, the particular coordinate system, and an "absolute" factor, the nature of the space-time continuum. The latter depends on local properties (intrinsic links) of space and time as well as on constraints imposed by the environmental phenomenon itself (physical law governing the attribute of interest, boundary and initial conditions, and attribute randomness sources). A summary outline of the space-time metric determination approach used in the present work is given in Figure 3.

The choice of the spatiotemporal metric has major consequences in the scientific modeling of environmental attributes. One consequence is related to the choice of mathematical models that establish linkages between spatiotemporally distributed data, such as covariance functions. These functions need to satisfy certain permissibility criteria that depend on the space-time metric (Christakos 2000; Christakos and Papanicolaou, 2000). The commonly used Gaussian function, e.g., is permissible in the case of a Euclidean metric but not in the case of a Manhattan metric. The exponential function, on the other hand, is permissible for both metrics. In general, the permissibility of a covariance function with respect to one metric form (e.g., Euclidean) does not guarantee its permissibility for another metric form (non-Euclidean). Space-time estimation, simulation, and mapping of environmental attributes depend on the metric assumed, since the covariance functions are used as inputs in environmental mapping and simulation techniques (e.g., Kriging, regression, and Kalman filtering). Hence, the same dataset can lead to different attribute maps if estimation or simulation is performed using different metrics.

There are a number of directions for future work concerning metric determination in environmental modeling, including the following: $(i)$ Different kinds of space/time-dependency of the metric coefficients could be studied, considering in particular those that correspond to physically meaningful space-time links. A classification of space/time-dependent metrics would be interesting too. Metric coefficients could be expressed in terms of standard analytic functions (polynomials, trigonometric and hyperbolic functions) of space-time coordinates. One may proceed by imposing symmetry or separability conditions on the metric, restricting the mathematical structure of the covariance function, or by considering the effects of 
BIC and random sources. (ii) Knowing the space-time metric in closed form, elucidation of its environmental properties is based on the phenomenon features. Certain metric solutions may not necessarily have a unique interpretation, which an issue in need of further investigation. (iii) If a metric form shows some resemblance to a known one, it should be investigated if the former can be reduced to the latter by means of a coordinate transformation, and what this means as regards the mathematical and physical interpretation of the $L_{G}$-equation. (iv) Another issue of interest is space-time coordinate transformation, i.e., while the $L_{G}$-equations is introduced in terms of a certain coordinate basis, coordinate transformation may be a possibility if it makes the $L_{G}$-equations more tractable and easier to solve.

Acknowledgements: This work has been supported by grants from the National Science Foundation of China (Grant No. NSFC 41671399), the Spanish-European Union MINECO/FEDER (Grant Nos MTM20 12-32666 and MTM2015-70840-P), and the Taiwan Ministry of Science and Technology (Grant Nos. MOST105-2221-E-002-039 and MOST105-2625-M-002-007).

\section{References}

Arkin, P.A., and Ardanuy, P.E. (1989). Estimating climatic-scale precipitation from Space: A review. J. Clim., 2, 1229-1238. https://doi. org/10.1175/1520-0442(1989)002<1229:ECSPFS >2.0.CO;2

Billings, S.D., Rick, K., Beatson, R.K., and Newsam, G.N. (2002). Interpolation of geophysical data using continuous global surfaces. Geophysics, 67(6), 1810-1822. https://doi.org/10.1190/1.1527081

Carroll, S. (2004). Spacetime and Geometry, Addison-Wesley, San Francisco.

Christakos, G., and Hristopulos, D.T. (1998). Stochastic Environmental Health Modeling, Kluwer Acad Publ., Boston.

Christakos, G. (2000). Modern Spatiotemporal Geostatistics, Oxford University Press, New York.

Christakos, G., and Papanicolaou, V. (2000). Norm-dependent covariance permissibility of weakly homogeneous spatial random fields. Stochastic Environ. Res. Risk Assess., 14(6), 471-478. https://doi. org/10.1007/s004770070001

Christakos, G., Hristopulos, D.T., and Bogaert, P. (2000). On the physical geometry hypotheses at the basis of spatiotemporal analysis of hydrologic geostatistics. Adv. Water Resour., 23, 799-810. https://doi.org/10.1016/S0309-1708(00)00020-8

Curriero, F.C. (2006). On the use of non-Euclidean distance measures in Geostatistics. Math. Geol., 38(8), 907-926. https://doi.org/10.10 07/s11004-006-9055-7

Dagan, G. (1989). Flow and Transport in Porous Formations, Springer, New York, NY. https://doi.org/10.1007/978-3-642-75015-1

Daley, R. (1999). Atmospheric Data Analysis, Cambridge Univ. Press, New York, N.Y.

Dobrovolski, S., 2010. Stochastic Climate Theory: Models and Applications, Springer, New York, NY.

Frei, C. (2014). Interpolation of temperature in a mountainous region using nonlinear profiles and non-Euclidean distances. Int. J. Climatol., 34(5), 1585-1605. https://doi.org/10.1002/joc.3786

Hadsell, F., and Hansen, R. (1999). Tensors of Geophysics, Vol. 2: Generalized Functions and Curvilinear Coordinates, Society of Exploration Geophysicists, Tulsa, OK.

He, X., Koch, J., Sonnenborg, T.O., Jorgensen, F., Schamper, C., and
Refsgaard, J.C. (2014). Transition probability-based stochastic geological modeling using airborne geophysical data and borehole data. Water Resour. Res., 50(4), 3147-3169. https://doi.org/10.1 002/2013WR014593

Jin, B., Wu, Y., Miao, B., Wang, X.L., and Guo, P. (2014). Bayesian spatiotemporal modeling for blending in situ observations with satellite precipitation estimates. J. Geophys. Res. (D Atmos.), 119(4), 1806-1819. https://doi.org/10.1002/2013JD019648

Klyatskin, V.I. (2015). Stochastic Equations: Theory and Applications in Acoustics, Hydrodynamics, Magnetohydrodynamics, and Radiophysics, Vol 1, Springer, New York.

Kyriakidis, P.C., and Journel, A.G. (1999). Geostatistical space-time models: A review. Math. Geol., 31(6), 651-684. https://doi.org/10. 1023/A: 1007528426688

Le, N.D., and Zidek, J.V. (2006). Statistical Analysis of Environmental Space-Time Processes, Springer, New York.

Lin, Y.C., Chang, T.J., Lu, M.M., and Yu, H.L. (2015). A space-time typhoon trajectories analysis in the vicinity of Taiwan. Stochastic Environ. Res. Risk Assess., 29(7), 1857-1866. https://doi.org/10. 1007/s00477-014-1001-5

Lloyd, C.D. (2010). Local Models for Spatial Analysis, CRC Press, Boca Raton. https://doi.org/10.1201/EBK1439829196

Monin, A.S., and Yaglom, A.M. (1971). Statistical Fluid Mechanics, M.I.T. Press, Cambridge.

Nieves, V., Llebot, C., Turiel, A., Sole, J., Garcia-Ladona, E., Estrada, M., and Blasco, D. (2007). Common turbulent signature in sea surface temperature and chlorophyll maps. Geophys. Res. Lett., 34(23). https://doi.org/10.1029/2007GL030823

Purser, R.J., Wu, W.S., Parrish, D.F., and Roberts, N.M. (2003). Numerical aspects of the application of recursive filters to variational statistical analysis. Part II: Spatially inhomogeneous and anisotropic general covariances. Mon. Weather Rev., 131(8), 1536-1548. https://doi.org/10.1175//2543.1

Reyes, J.M., and Serre, M.L. (2014). An LUR/BME framework to estimate PM2.5 explained by on road, mobile and stationary sources. Environ. Sci. Technol., 48(3), 1736-1744. https://doi.org/10.1021/ es4040528

Soong, T.T. (1973). Random Differential Equations in Science and Engineering. Acad. Press, London, UK.

Srinivasan, S.K., and Vasudevan, R. (1971). Introduction to Random Differential Equations and their Applications. Amer. Elsevier Publ. Co., Inc, New York, NY.

Stein, A., Groenigen J.W.V., Jeger, M.J., and Hoosbeek, M.R. (1998). Space-time statistics for environmental and agricultural related phenomena. Environ. Ecol. Stat., 5(2), 155-172. https://doi.org/ 10.1023/A:1009614821618

Stephani, H., Kramer, D., MacCallum, M., Hoenselaers, C., and Herlt, E. (2003). Exact Solutions of Einstein's Field Equations, Cambridge, UK. https://doi.org/10.1017/CBO9780511535185

Tartakovsky, D.M., and Broyda, S. (2011). PDF equations for advective-reactive transport in heterogeneous porous media with uncertain properties. J. Contam. Hydrol., 120-121, 129-140. https://doi. org/10.1016/j.jconhyd.2010.08.009

Taylor, G.I. (1938). The spectrum of turbulence. Proc. of the Royal Society A, 164, 476-490. https://doi.org/10.1098/rspa.1938.0032

Turcotte, D.L. (1997). Fractals and Chaos in Geology and Geophysics, Cambridge University Press, Cambridge, UK. https://doi.org/ 10.1017/CBO9781139174695

Zhang, D. (2002). Stochastic Methods for Flow in Porous Media, Academic Press, San Diego, CA.

Zhu, Z., Li, T., Hsu, P.C., Jinhai, He, J. (2015). A spatial-temporal projection model for extended-range forecast in the tropics. Clim. Dyn., 45, 1085-1098. https://doi.org/10.1007/s00382-014-2353-8 